\title{
Sister Joseph Nodule the Durable Sign
}

Amer Hashim Al Ani ${ }^{1^{*}}$, Ehab Totah ${ }^{2}$ and Hashim Amer Al Ani ${ }^{3}$

${ }^{1}$ Department of General Surgery, Bahrain specialist Hospital, Manama, Bahrain

${ }^{2}$ Department of General Surgery, Al-Bashir, Teaching Hospital, Amman, Jordan

${ }^{3}$ Medical School, Jordanian University, Amman, Jordan

"Corresponding author: Dr. Amer Hashim Al-Ani, MB ch B, CABS, FICM "digestive surgery", FJCMS, FRCS ( Glasgow), Department of General Surgery, Bahrain Specialist Hospital, Juffair Manama, Bahrain, Tel: 00962788266034; E-mail: ameralqadi2002@yahoo.com

Rec date: Nov 13, 2014 Acc date: April 16, 2015 Pub date: April 20, 2015

Copyright: $\odot 2015 \mathrm{Al}-\mathrm{Ani} \mathrm{AH}$, et al. This is an open-access article distributed under the terms of the Creative Commons Attribution License, which permits unrestricted use, distribution, and reproduction in any medium, provided the original author and source are credited.

\section{Abstract}

Introduction: Sister Mary Joseph sign, refers to an umbilical nodule in individuals who invariably had a poor outcome and succumbed relatively early, due to metastasis of a malignant cancer in the pelvis, or abdomen.

Presentation of case: We report here a 39 year old male presented with abdominal distension, night sweating, anorexia, and weight Loss. On examination a hard nodule in the umbilical region (Sister Mary Joseph sign) was discovered. CTscan showed omentum cake; ascites and umbilical nodule .colonoscopy showed upper rectal tumor .diagnostic laparoscopy and biopsy confirm metastatic adenocarcinoma disseminated intraperitonealy. Despite the chemotherapy, he died after two months.

Discussion: Sister Joseph sign is usually represents an advanced disease, and carries a very poor prognosis. Because the nodule is so easy to biopsy and because most associated cancers are inoperable at the time of diagnosis, the ability to identify this lesion may save a patient an unnecessary diagnostic surgery.

Conclusion: Clinical examination, (in the presence of high technology investigation), is still of high value in the diagnosis.
\end{abstract}

Keywords: Umbilical nodule, Sister Joseph sign, Abdominal distension, Night sweating, Anorexia, Weight loss

\section{Introduction}

Lesions in the umbilicus can be either benign or malignant. A malignant umbilical mass can represent a primary or metastatic lesion. Sister Mary Joseph sign, refers to a palpable nodule bulging into the umbilicus as a result of metastasis of a malignant cancer in the pelvis or abdomen [1,2]. Sister Mary Joseph Dempsey (1856-1939) was the surgical assistant of William J. Mayo at St. Mary's Hospital in Rochester, Minnesota from 1890 to 1915. She drew Mayo's attention to the presence of a firm mass at the umbilicus in individuals who invariably had a poor outcome and succumbed relatively early postoperatively. William J. Mayo published an article about it in 1928. The eponymous term Sister Mary Joseph nodule was coined in 1949 by Hamilton Bailey [2].

\section{Case Report}

39 year old male was admitted with complaints of abdominal distension, Night sweating, Anorexia, weight loss, for the last two months. There was no abdominal pain, no nausea, no vomiting. He had normal bowel motion. There was no fever. His past medical, surgical, drug and family history was not significant. He is Smoker (20 cig/D/20 years).

On examination the patient was obese, not pale, not jaundiced .His abdomen was distended, with striae, ascites and a hard nodule in the umbilical region (Figure 1). Digital rectal examination was negative for masses, or blood in stool. There was no leg edema.

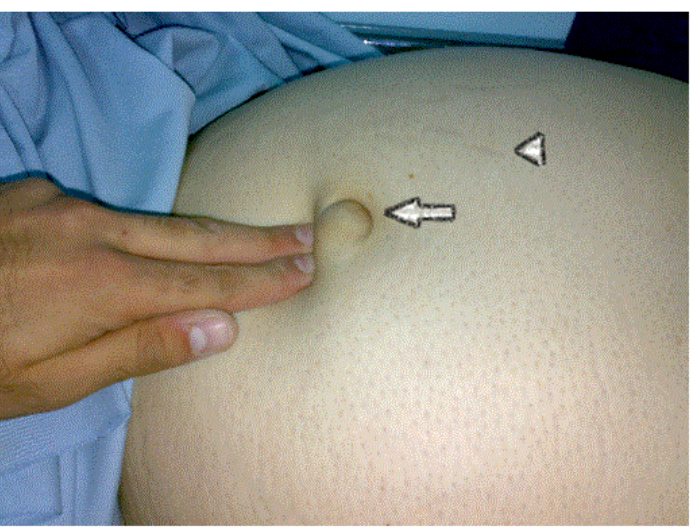

Figure 1: The abdomen was distended, with striae (arrow head), ascites and a hard nodule (arrow) in the umbilical region.

All his laboratory blood tests were within normal, including levels of carcinoembryonic antigen, carbohydrate antigen 19-9, and cytokeratin 19 fragment. Abdomen CT scan showed: Ascites, omental cake and nodule in the umbilicus (Figure 2).

Upper gastrointestinal endoscopy was unremarkable. Colonoscopy revealed upper rectal mass, its biopsy showed adenocarcinoma. Ascitic 
Page 2 of 3

fluid aspiration was done. The fluid examination was negative for acid fast bacilli. WBCs count was 320/L (75\% mono nuclear cells), RBCs count was: 832/L. the Albumin: was $63.10 \mathrm{~g} / \mathrm{L}(30.50 \mathrm{~g} / \mathrm{L})$. The Ascitic fluid was exudates. Cytology examination showed: Many lymphocytes $90 \%$, few reactive mesothelial cells, and occasional clusters of cells with enlarged irregular hyperchromatic neuclei, suspicious for malignancy.

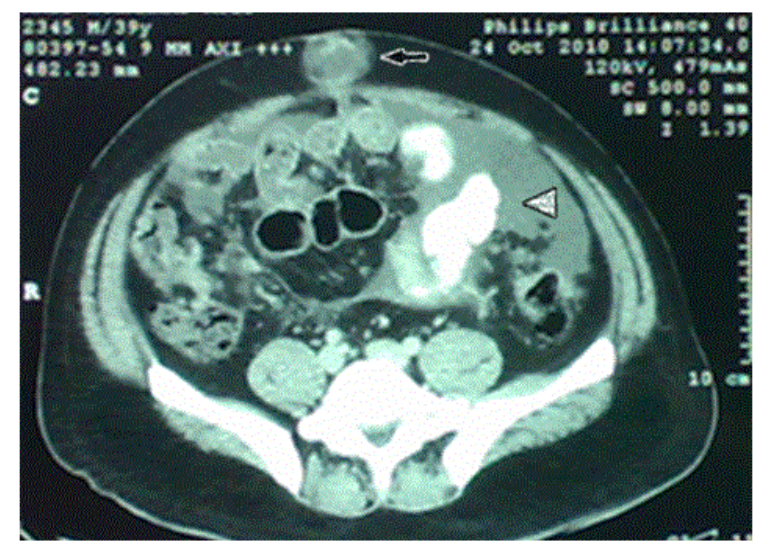

Figure 2: Abdominal CT scan showed: Ascites (arrow head), and nodule in the umbilicus (arrow).

Diagnostic laparoscopy showed (Figure 3) Ascites, Multiple fibrinous adhesions and multiple whitish nodules (covering the liver, peritoneal cavity, and falciform ligament). Biopsies were taken. The Histopathology result showed metastatic adenocarcinoma in all mentioned sites .The adenocarcinoma was of moderate differentiation, with small foci of extracellular mucin secretion .Immune stains for CK7, CK 20 were positive, and for TTFI were focally positive.

Patient was sent for Neoadjuvant therapy. 2 months later he died.

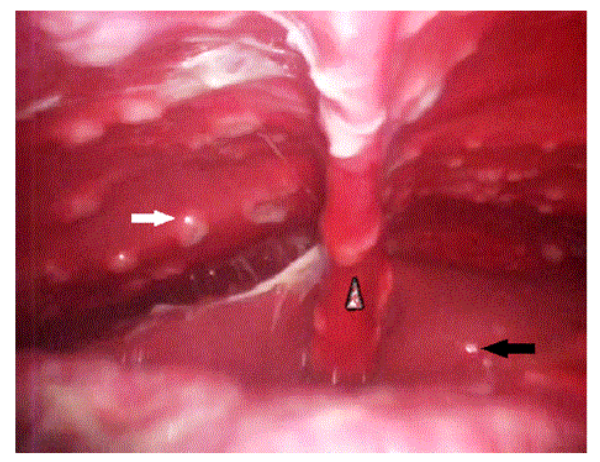

Figure 3: Diagnostic laparoscopy showed multiple nodules on the liver (black arrow), peritoneal cavity (white arrow) and falciform ligament (arrow head).

\section{Discussion}

Malignant tumors of the umbilical region make up about $43 \%$ of all umbilical tumors. Primary malignant umbilical tumors can be melanomas, basal cell carcinomas, squamous cell carcinomas, myosarcomas, and adenocarcinomas; primary tumors of the umbilical region are exceedingly rare, constituting only $17 \%$ of malignant umbilical tumors. 1 Metastatic tumors of the umbilical region, constituting $83 \%$ of all malignant umbilical tumors $[1,3]$.

Sister Mary Joseph sign, refers to a palpable nodule bulging into the umbilicus as a result of metastasis of a malignant cancer in the pelvis or abdomen $[1,4]$. In this case the tumor was Metastatic tumors of the umbilical region from rectal tumor, it was adenocarcinoma. It is estimated that 1 to $3 \%$ of patients with abdomino-pelvic malignancy could present with a Sister Mary Joseph's nodule [5].

Epidemiological studies revealed that Sister Mary Joseph's nodule predominates in women [5]. In this case the patient was male. The mean age of patients at the time of diagnosis ranges from 18-87 years [6]. The patient in this case is within the usual range (39 years). Gastrointestinal tract is the most common location of the primary neoplasm in Sister Mary Joseph sign (35 to 65\%), followed by a gynecological origin (12 to 35\%) [7]. The common sites in decreasing order of frequency are: stomach (25\%), colorectal (10\%), and pancreas (7\%) [6,7]. In females, ovarian cancer is the most common primary site, of which serous papillary cystadenocarcinoma is the most frequent (34\%) [3,7].

Primary tumors in many other sites have been reported to lead to Sister Mary Joseph's nodule, including gallbladder, liver, breast, lung, prostate, penis, peritoneum, lymphoma, bladder, kidney, endometrium, cervix, vagina, vulva, and fallopian tube [3,6]. In 15 to $30 \%$ of the cases, the source of the primary site of the tumor is unknown [8].

The tumor may spread to the umbilicus through the lymph ducts or blood vessels, by contiguous extension, or through embryologic remnants [9]. Patients with Sister Mary Joseph's nodule often present with a painful lump with irregular margins and hard consistency. The surface may be ulcerated and necrotic with either blood, serous, purulent, or mucous discharge from it [7]. In this case, the patient was complaining from abdominal distension (he did not notice the nodule). Various imaging modalities can aid in establishing the diagnosis, such as ultrasonography, CT, MRI, and PET [10]. Once Sister Mary Joseph's nodule is discovered, a biopsy - either excisional or fine needle aspiration cytology - is mandatory to establish diagnosis and to find the possible primary site [11]. The histopathological evaluation may show characteristics of the underlying tumor, while immunohistochemical marker studies and ultrastructural examination may help delineate the tissue of origin $[12,13]$. In this case diagnostic laparoscopy was done to prove the relation between sister Joseph sign and advanced intraabdominal malignancy .Sister Mary Joseph's nodule usually represents widespread metastasis $[14,15]$ and treatment is commonly palliative [10]. Several authors have advocated wide excision with extensive search for the primary lesion, radiotherapy and surgery with adjuvant therapy $[10,15]$,. patients treated aggressively with both surgery and adjuvant therapy live for an average of 17.6 months, which is more than with surgery alone (7.4 months), adjuvant therapy alone (10.3 months), or no treatment (2.3 months) [11,15,16]. An aggressive surgical approach combined with chemotherapy may improve survival $[11,15]$. In this case after discussion with the patient and his family, they choose the chemotherapy as a mode of treatment (as they understand that the treatment is palliative, and they try to avoid the side effects of the aggressive treatment). 


\section{Conclusion}

Sister Joseph's nodule is the best example of importance of a careful methodological examination in the era of sophisticated imaging studies. It often indicates advanced, metastatic intra-abdominal cancer. Biopsy can easily be harvested and aid in confirming the diagnosis, planning treatment and expecting prognosis.

\section{References}

1. Yendluri V, Centeno B, Springett G (2007) Pancreatic cancer presenting as a Sister Mary Joseph's nodule: case report and update of the literature. Pancreas 34: 161-164.

2. Bailey H (1949) Demonstration of physical signs in clinical surgery. 11th edition, Baltimore, Williams \& Wilkins 227.

3. Panaro F, Andorno E, Di Domenico S, Morelli N, Bottino G, et al. (2005) Sister Joseph's nodule in a liver transplant recipient: case report and mini-review of literature. World J Surg Oncol 3: 4.

4. Trebing D, Göring HD (2004) The umbilical metastasis. Sister Mary Joseph and Her time [in German]. Hautarzt 55: 186-189.

5. Kolwijck E, Boss EA, van Altena AM, Beex LV, Massuger F (2007) Stage IV epithelial ovarian carcinoma in an 18 year old patient presenting with a Sister Mary Joseph's nodule and metastasis in both breasts: a case report and review of the literature. Gynecol Oncol 107: 583-585.

6. Al-Mashat F, Sibiany AM (2010) Sister Mary Joseph's nodule of the umbilicus: is it always of gastric origin? A review of eight cases at different sites of origin. Indian J Cancer 47: 65-69.

7. Gabriele R, Conte M, Egidi F, Borghese M (2005) Umbilical metastases: current viewpoint. World J Surg Oncol 3: 13.
8. Gabriele R, Borghese M, Conte M, Basso L (2004) Sister Mary Joseph's nodule as a first surgery of cancer of the caecum: report of a case. Dis Colon Rectum 47: 115-117.

9. Piura B (2006) [Umbilical metastasis: Sister Mary Joseph's nodule]. Harefuah 145: 505-550.

10. Chau CL, Griffith JF (2005) Musculoskeletal infections: ultrasound appearances. Clin Radiol 60: 149-159.

11. Handa U, Garg S, Mohan H (2008) "Fine-needle aspiration cytology of Sister Mary Joseph's (paraumbilical) nodules,” Diagnostic Cytopathology 36: $348-350$.

12. Ioannidis AC, Stavrakis T (2010) "SisterMary Joseph's nodule as the sole presenting sign of gastric signet ring cell adenocarcinoma," Gastroenterologie Clinique et Biologique 34: 565-568.

13. Powell JL (2011) "Powell's pearls: eponyms in medical and surgical history. Sister Joseph's nodule; Sister Mary Joseph (1856-1939),” Journal of Surgical Education 5: 442 - 443.

14. Papalas JA, Selim MA (2011) "Metastatic vs primary malignant neoplasms affecting the umbilicus: clinicopathologic features of 77 tumors," Annals of Diagnostic Pathology 15: 237-242.

15. Kurt S, Camuzcuoglu H, Camuzcuoglu A, Dicle N, Tinar S (2009) "Umbilical metastasis of serous component as a first sign of mixed type epithelial ovarian cancer," European Journal of Gynaecological Oncology 30: 455-457.

16. Majmudar B, Wiskind AK, Croft BN, Dudley AG (1991) The Sister Mary Joseph nodule: its significance in gynecology. Gynecol Oncol 40: 152159. 